\title{
The neural network-based control system of direct current motor driver
}

\author{
Trong-Thang Nguyen \\ Faculty of Energy Engineering, Thuyloi University, Vietnam
}

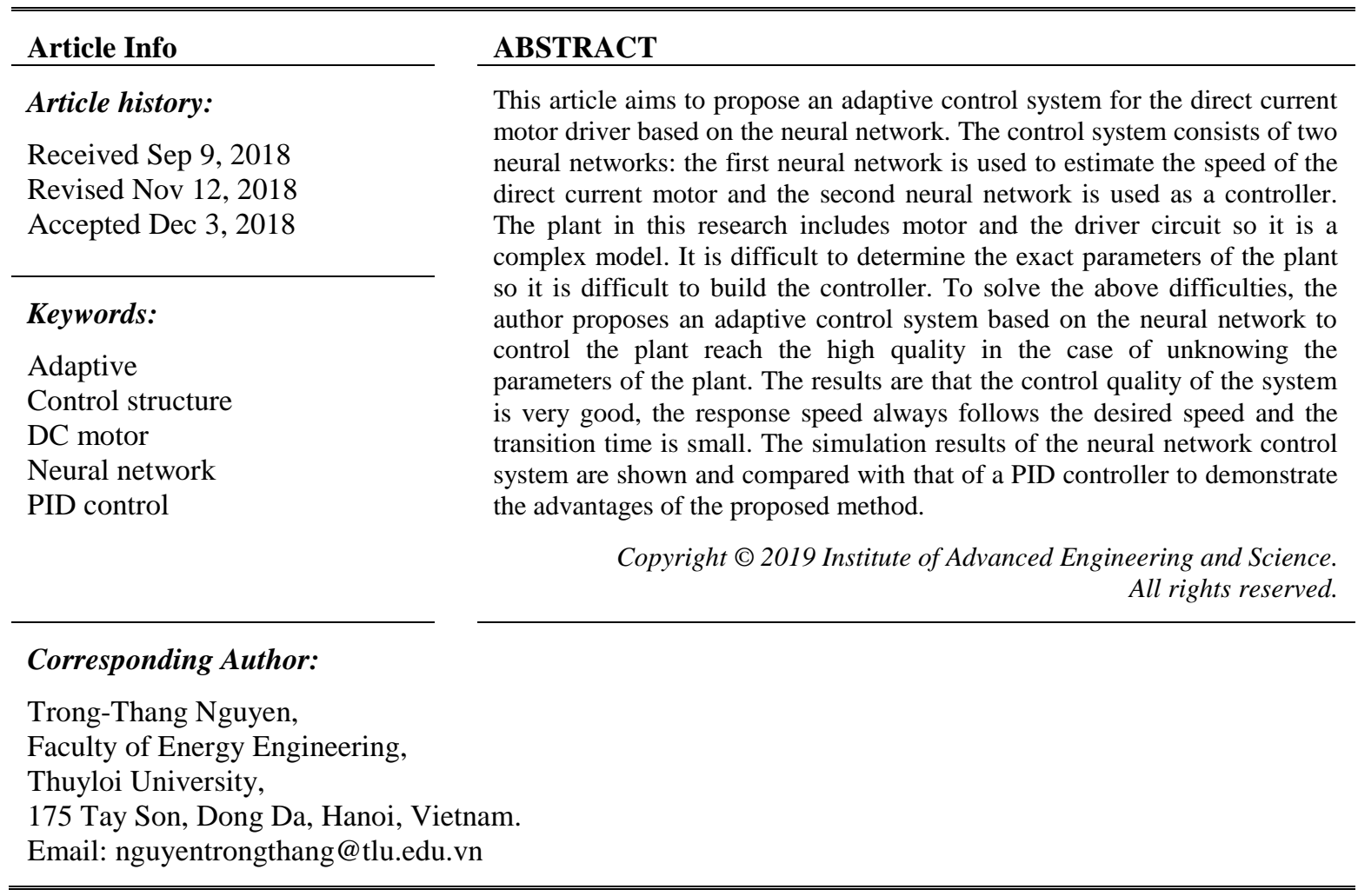

\section{INTRODUCTION}

The Direct Current (DC) motor is one of a traditional electric motor. In comparison to the other electric motor such as brushless DC motors [1], induction machine [2], [3], the DC motor has superior features such as the large electromagnetic torque, the simple control structure and the adjust ability of the speed in a wide range. Thus, the DC motor is still very popular in the application of industrial areas required the high-quality motion, such as mining, transportation, steel rolling, etc. So, the performance development of motor applications is very important. There are many research for controlling DC Motors such as [4]-[7], the most common method is using the Proportional-Integral-Derivative (PID) controllers. However, in several working modes of the DC motor, the characteristics of the DC motor are nonlinear so the PID controller is not suitable, the quality control is low.

There is some research about controlling the nonlinear objects such as the backstepping control method [8], the sliding mode control method [9], the linearization technique [10], etc. The limitations of the above methods are that the difficult problem with the choice of appropriate Lyapunov function and the chattering phenomenon's existence. There is a solution to control the DC motor that has achieved highquality, such as the control method based on the flatness principle [11]. However, the limitation of this solution is the need of knowing exactly the parameters of the DC motor.

To overcome all the limitations of the previous method, in this study, the author proposes a solution to build a control system based on the Neural Network of Model Reference Adaptive Architecture. The advantages of control systems using neural network are that the system can control both linear and nonlinear 
objects. The system can work well without knowing the parameters even without knowing the structure of the plant. The controller can adjust, to adapt itself to the different status of the plant.

\section{BUILDING THE DRIVER CIRCUIT}

\subsection{The structure and the equation of the DC motor}

The diagram of a separately excited DC Motor is presented as Figure 1 [11], it includes:

a. The field windings are in the stator, they are used to excite the field flux.

b. The armature coils are on the rotor, they are supplied current via brush and the commutator.

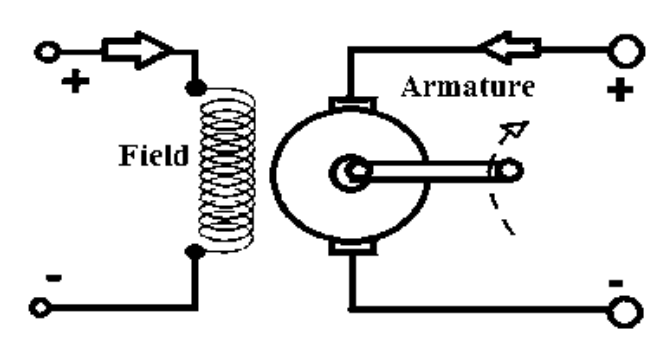

Figure 1 . The diagram of a separately excited DC Motor

The mathematical equations of DC Motors are as follows:

c. The voltage equation:

$$
u_{a}=R_{a} i_{a}+L_{a} \frac{d i_{a}}{d t}+E(\mathrm{~V})
$$

Where $u_{a}$ is the armature voltage; $R_{a}, L_{a}$ is the armature resistance and inductance.

d. The electromotive equation:

$$
E=K_{E} \omega=\left(L_{a f} \cdot i_{f}\right) \cdot \omega(\mathrm{V})
$$

Where $\omega$ is the speed of the rotor, $K_{E}$ is the coefficient of voltage, $i_{f}$ is the winding field current, $L_{a f}$ is the field armature mutual inductance.

e. The motion equation:

$$
J \frac{d \omega}{d t}=T_{e}-T_{L}-B_{m} \omega-T_{f}(\mathrm{~N} . \mathrm{m})
$$

Where $J$ is the inertia, $T_{e}$ is the electromechanical torque, $T_{L}$ is the torque which impact to the shaft, $B_{m}$ is the coefficient of the viscous friction, $T_{f}$ is the coulomb friction torque.

f. The electromechanical torque equation:

$$
T_{e}=K_{T} i_{a}=\left(L_{a f} \cdot i_{f}\right) \cdot i_{a}(\mathrm{~N} . \mathrm{m})
$$

Where $K_{T}$ is the coefficient of the torque.

\subsection{Building the power circuit}

The field winding is connected to a constant DC voltage source. To control the motor speed, we only need to change the current supplied to the armature coil of DC motor, according to (4), the 
electromechanical torque (Te) will be changed. According to (3), if the electromechanical torque Te is changed, the motor speed is changed.

In addition, to increase the flexibility, the DC motor must be able to reverse the rotation. So, the power circuit is connected in the $\mathrm{H}$ form, it has four switches Q1-Q4. If we need the DC motor rotates right, we control Q1 and Q4 are on, Q2 and Q3 are off. Conversely, if we need the DC motor rotates left, we control Q1 and Q4 are off, Q2 and Q3 are on. The power circuit of the DC motor is shown in Figure 2.

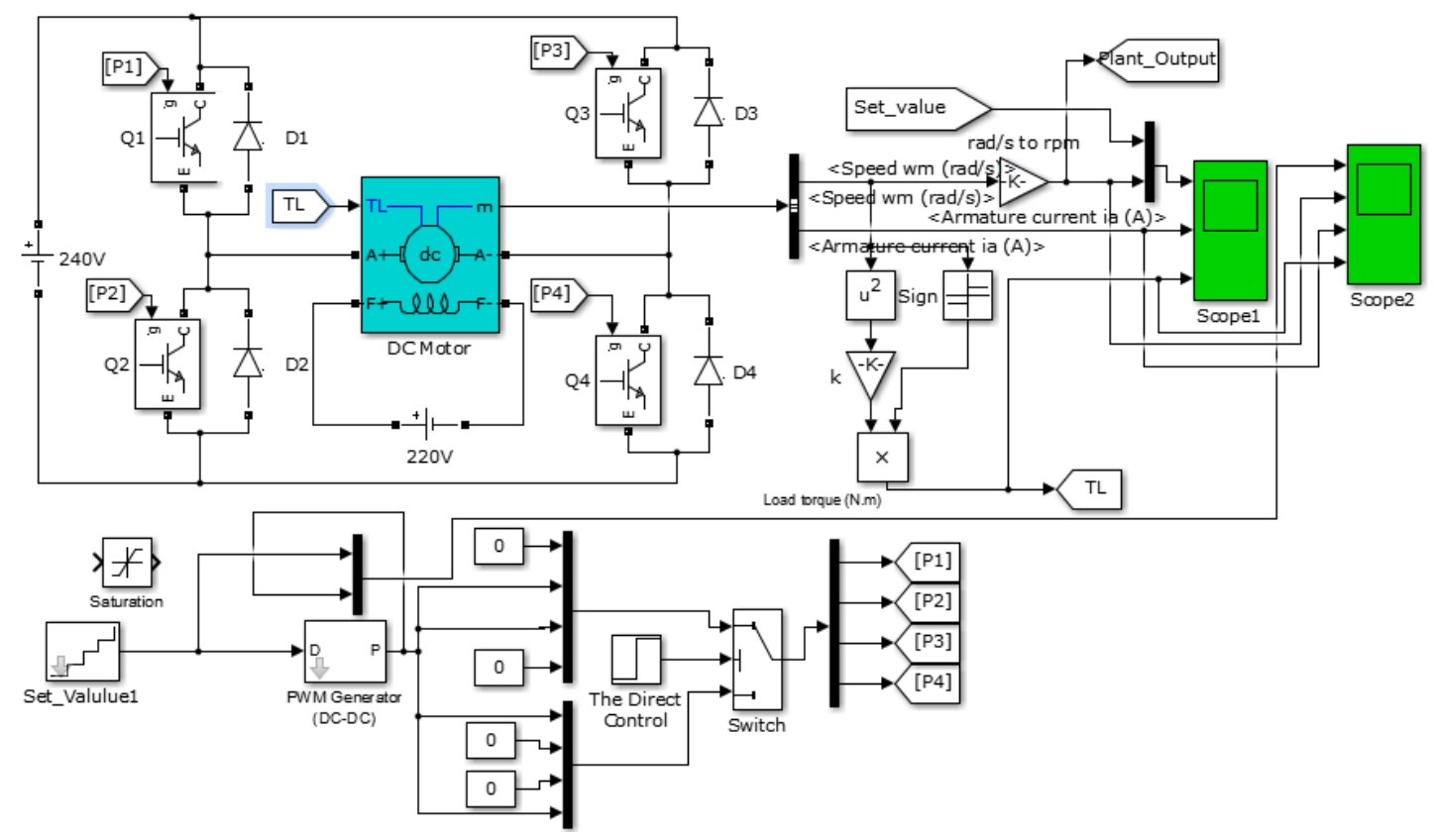

Figure 2. The power circuit of the DC motor

In the power circuit, to change the current fed into the armature coil $i_{a}$, we change the pulse fed into the switches based on Pulse-Width Modulation (PWM) method. The PWM is a method, which is that using a rectangular pulse wave with the value of width $(T)$ is variable and the value of duty cycle $(D)$ is constant. The graph example of the PWM modulation is shown in Figure 3.

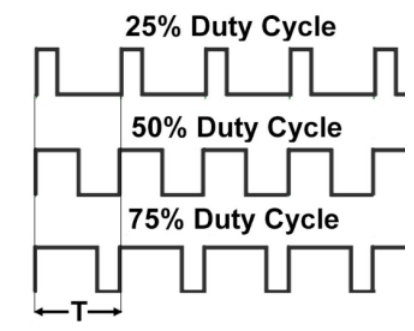

Figure 3. The graph of the PWM modulation

\section{BUILDING THE CONTROL SYSTEM BASED ON THE NEURAL NETWORK}

\subsection{The control object}

On the basis of the power control circuit, we define the control object, the input is the value of width pulse with the range value is $(0-1)$, the output is the motor speed. To represent the relationship between the input and the output of the control object, we run the system in the case the pulse width value changes from 0.6 to 1 , the result is shown in Figure 4.

The Figure 4 shows that if the pulse width increases, the motor speed increases and if the pulse width decreases, the motor speed decreases. However, the relationship between the pulse width (the input of 
the control object) and the speed (the output of the control object) is a complex relationship so it is difficult to determine the exact equation of this relationship. Thus, the author proposes the control solution based on neural network, because the neural network controller is highly adaptable and self-adjust fit the status of the control object.

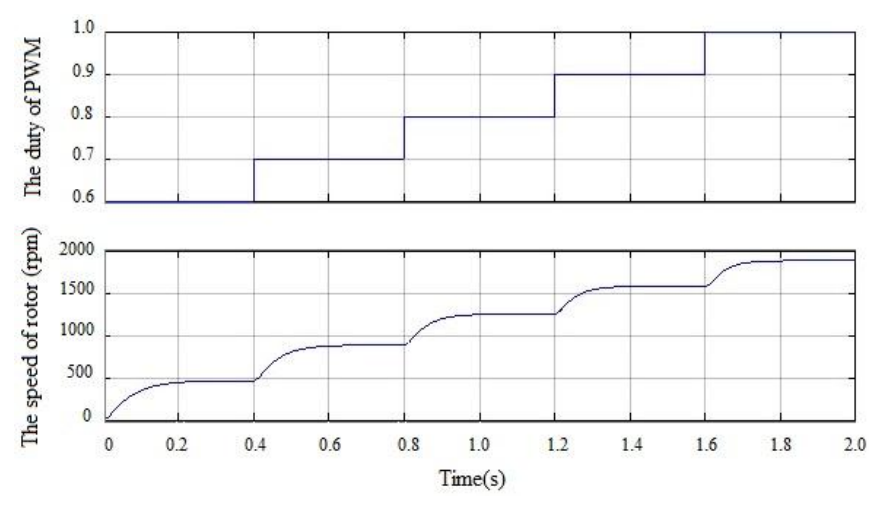

Figure 4. The the graph of the pulse width and the motor peed

\subsection{The model reference control system using neural network}

In the dynamic systems, the disturbance, friction and unknown parameter are the main reasons to reduce the control quality. To solve that problem, in this research, the author builds an artificial neural network for controlling the system. A neural network includes many simple components, these components are the single-input neuron or the Multiple-Input Neuron, are shown as the Figure 5 [12].

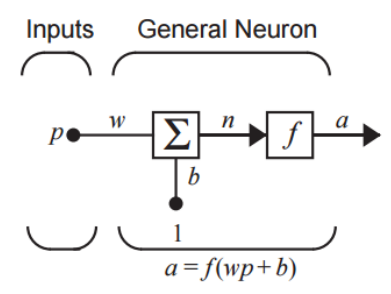

a)

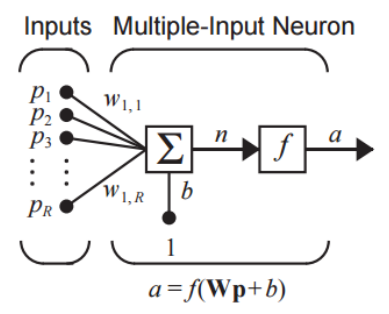

b)

Figure 5. The component of neural network: (a) the case of single-input, (b) the case of multiple-input

In the case of singer-input, the output of the neuron is calculated as (5):

$$
a=f(w \cdot p+b)
$$

Where $p$ is the scalar input, $w$ is the scalar weight, $b$ is the bias, $f$ is the transfer function, $a$ is the scalar neuron output. The scalar weight $w$ and the bias $b$ are adjusted by the learning rule in order for the relationship between the neuron input and neuron output meets the mission goal. The transfer function is a linear or a nonlinear function, the transfer function is chosen by the designer.

In the case of multiple-input, the neuron network includes $R$ inputs. Thus, $p$ is the vector which includes $R$ inputs $p_{l}, p_{2}, \ldots, p_{R}$. $\boldsymbol{w}$ is the weight matrix which includes $w_{l, l}, w_{1,2}, \ldots, w_{l, R}$. The diagram is shown in Figure 4(b), the output of the neuron network is calculated as (6):

$$
a=f(\boldsymbol{w} \cdot \boldsymbol{p}+b)
$$

Usually, a neural network has several layers. Each layer has a bias vector $(\boldsymbol{b})$ and a weight matrix $(\boldsymbol{w})$ and an output vector $(\boldsymbol{a})$. Example, Figure 6 shows the network neural with three layers, the outputs of 
the first layer is the input of the second layer, the outputs of second layer is the input of the third layer. The output layer is at once with whose output is the network output, the other layers are named hidden layer.

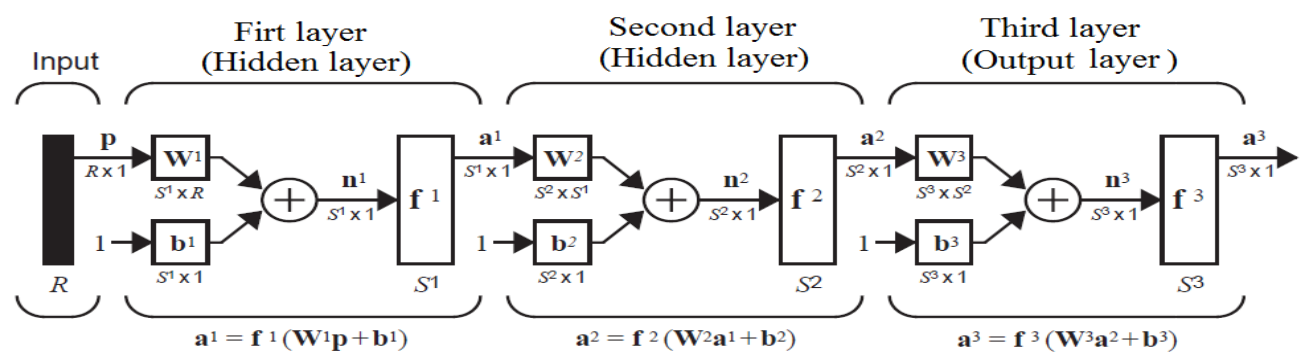

Figure 6. The neural network with three layers

A number of neural network control architectures have been proposed [13]-[15]. In this research, the author will use the Model Reference Control Architecture [16], [17] for controlling the driver circuit of DC motor. The control architecture is shown in the Figure 7. It is included two neural networks: the neural network controller and the neural network plant model.

The neural network controller creates the suitable control signal fed into the plant in order to control the plant output value as close as possible to the desired value. This desired value is determined by the reference model. The control error between the plant output value and the reference model output value is used in the adaptive algorithm to adjust the controller parameters.

In reality, the plant parameters are unknown, so the adaptive algorithm updates the controller parameters in order that the control error asymptotically equals zero. The detailed Model Reference Control Structure is shown in the Figure 8.

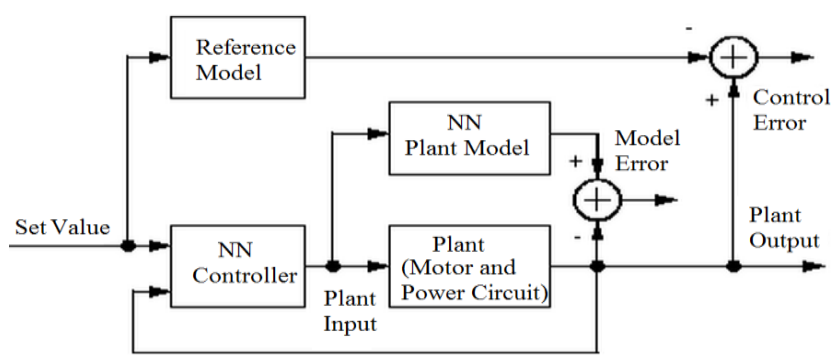

Figure 7. The model reference control architecture for controlling the driver of DC motor

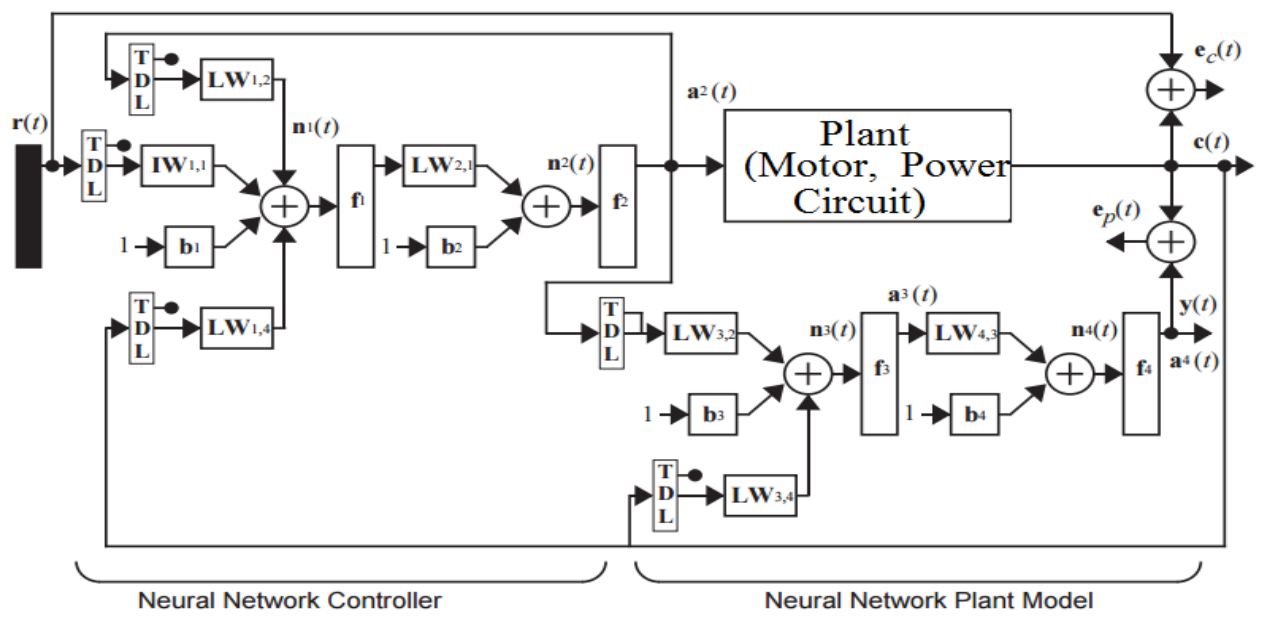

Figure 8 . The detailed model reference control structure 


\subsection{Training the neural network}

The control system of neural network includes two parts. The first one is the neural network of reference model which is used to estimate the motor speed. The second one is the neural network of controller, which is used to generate a control signal for controlling the plant (the converter).

To set the control system, firstly we must train the neural network plant model. After the plant model is identified, we train the neural network controller in order for the plant output follows the reference model output. In this research, we set these neural networks included two layers, the activation functions of the hidden layer is sigmoid function, the activation functions of the output layer is a linear function. These neural networks are trained by back-propagation update rules. The training results of the neural network are shown in Figure 9.
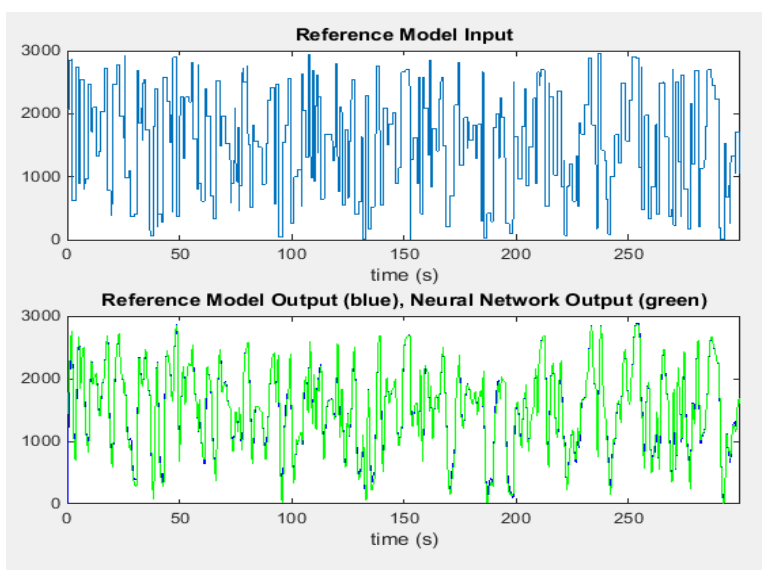

Figure 9. The training results of the neural network

\section{RESULTS AND ANALYSIS}

\subsection{The control schemes}

To demonstrate the advantage of the proposed neural network controller, we run the simulation in two cases: the PID controller, and the neural network controller. The control system structures are shown in Figure 10.

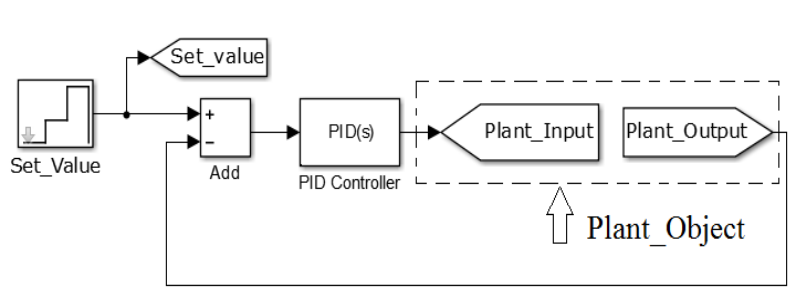

(a)

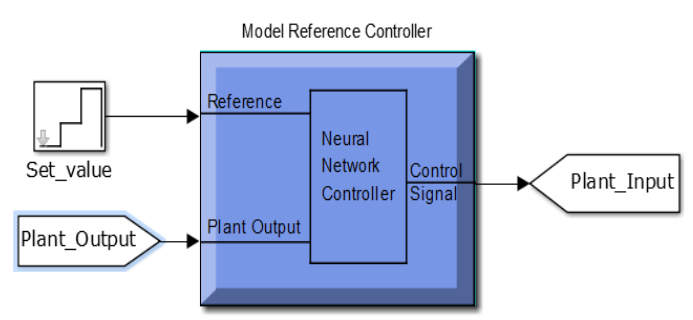

(b)

Figure 10. The control schemes: (a) in the case of PID controller, (b) in the case of neural network controller

The parameters of the PID controller are determined by the process of experiment and many times of the adjustment, the optimal parameters of the PID controller are as follows: $K p=0.02, K i=0.05, K d=0$. The DC motor parameters are shown in Table 1.

Table 1. The DC Motor Parameters

\begin{tabular}{llllllll}
\hline $\operatorname{Ra}(\Omega)$ & $\mathrm{La}(\mathrm{H})$ & $\operatorname{Rf}(\Omega)$ & $\mathrm{Lf}(\mathrm{H})$ & $\mathrm{Laf}(\mathrm{H})$ & $\mathrm{J}\left(\mathrm{kg} . \mathrm{m}^{\wedge} 2\right)$ & $\mathrm{Bm}(\mathrm{N} . \mathrm{m} . \mathrm{s})$ & $\mathrm{Tf}(\mathrm{N} . \mathrm{m})$ \\
\hline 2.61 & 0.031 & 272.5 & 161 & 1.12 & 0.031 & 0.0032 & 0.48 \\
\hline
\end{tabular}




\subsection{The simulation results}

In the case of the PID controller, the simulation results of the control system are shown in Figure 10. In the case of the neural network controller, the simulation results of the control system are shown in Figure 11. Each simulation result consists of the following characteristics: the desired speed, the actual speed of the DC motor. In addition, to express the objectivity of the simulation results, the simulation results also show the graph of the armature current, the load torque.

The simulation results show that: In the case of PID controller, the system has also met the control requirements. However, the quality of control is not as good. For example, the overshoot is high, the transition time is long. In the case of neural network controller, the quality of the system has improved a lot. The overshoot is low, the transition time is small. The simulation results in the case of neural network controller as shown in Figure 12.
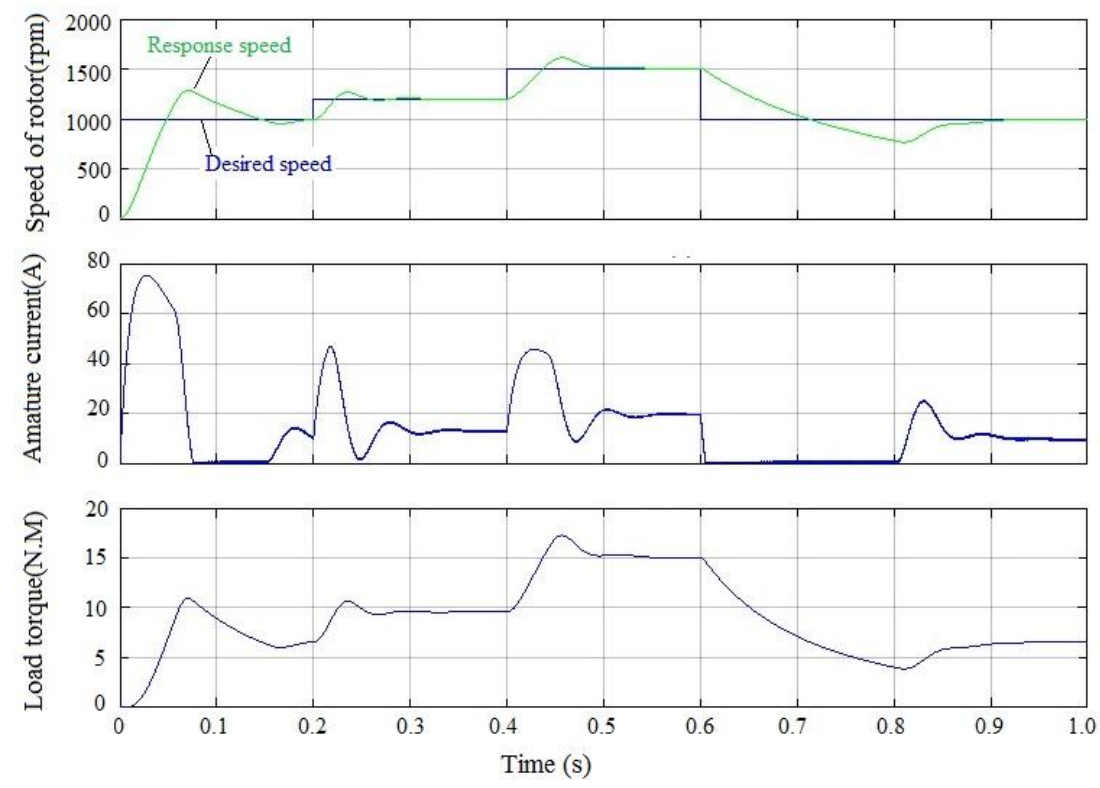

Figure 11. The simulation results in the case of PID controller
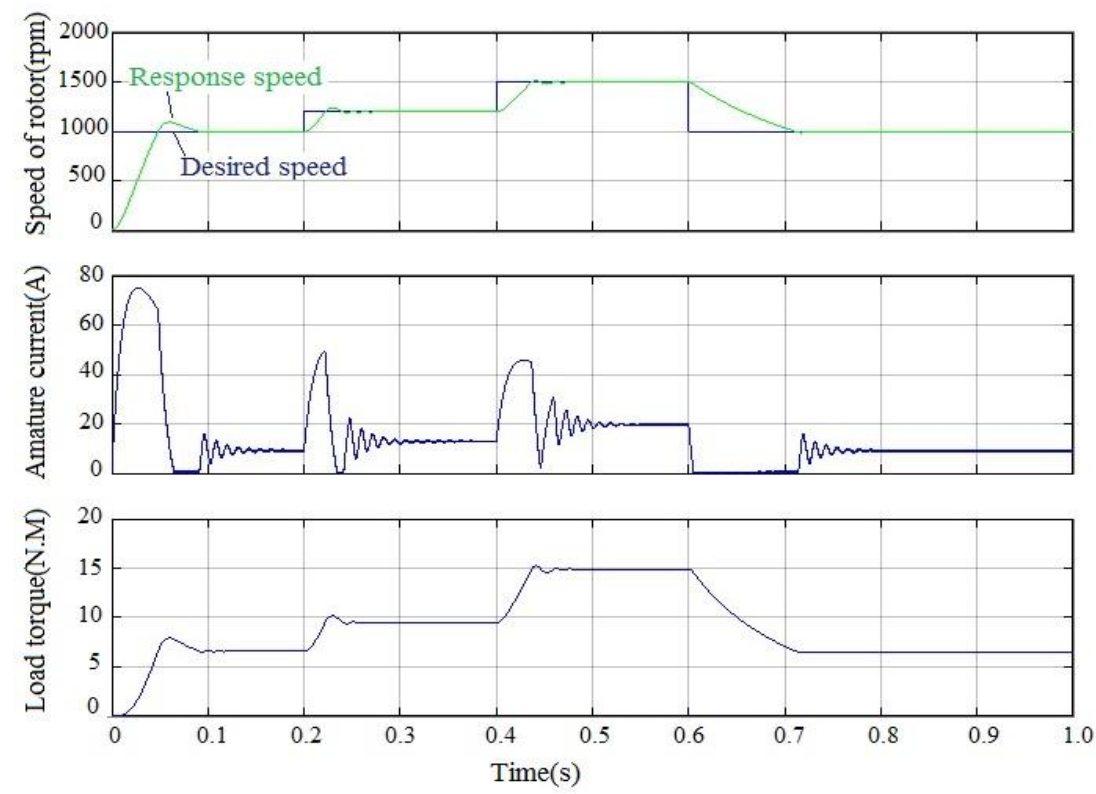

Figure 12. The simulation results in the case of neural network controller 


\section{CONCLUSION}

In this research, the author has succeeded in building the neural network- based model reference control system for controlling the driver circuit of the DC motor. The system has controlled the control object achieved the high-quality in the conditions without knowing the parameters and structure of the control object. The results have been verified through the simulation on Matlab and compared in the traditional PID controller. The results show that the quality of the proposed neural control system has been greatly improved. The response speed of the DC motor always follows the desired speed with the short transition time. The success of this research will be the basis for further research. In the next research, the author will install the neural network control algorithm on the microcontroller for controlling the real plant.

\section{REFERENCES}

[1] Park, J. S., \& Lee, K. D. "Design and Implementation of BLDC Motor with Integrated Drive Circuit,” International Journal of Power Electronics and Drive Systems (IJPEDS), 8(3), 1109-1116, 2017.

[2] Abdelhak, B., \& Bachir, B. "A High Gain Observer Based Sensorless Nonlinear Control of Induction Machine," International Journal of Power Electronics and Drive Systems, 5(3), 305, 2015.

[3] Gunabalan, R., \& Subbiah, V., "Speed Sensorless Vector Control of Induction Motor Drive with PI and Fuzzy Controller," International Journal of Power Electronics and Drive Systems, 5(3), 315, 2015.

[4] Bosco, Maycon Chimini, et al,"Estimation of Parameters and Tuning of a Speed PI of Permanent Magnet DC Motor using Differential Evolution," Electric Machines and Drives Conference (IEMDC), IEEE International, pp.1-6, 2017.

[5] Yao, Jianyong, Zongxia Jiao, and Dawei Ma,"Adaptive Robust Control of DC Motors with Extended State Observer," IEEE Transactions on Industrial Electronics, Vol 61.7, pp. 3630-3637,2014.

[6] Xue, Dingyu, Chunna Zhao, and YangQuan Chen, "Fractional Order PID Control of a DC-Motor with Elastic Shaft: A Case Study," American Control Conference, IEEE, pp.1-6, 2006.

[7] Thomas, Neenu, and Dr P. Poongodi, "Position Control of DC Motor using Genetic Algorithm Based PID Controller," Proceedings of the World Congress on Engineering, vol. 2, pp. 1-3, 2009.

[8] Trabelsi, R., Khedher, A., Mimouni, M. F., \& M'sahli, F. Backstepping Control for an Induction Motor using an Adaptive Sliding Rotor-flux Observer," Electric Power Systems Research, 93, 1-15, 2012.

[9] Abdelmadjid, G., Seghir, B. M., Ahmed, S., \& Youcef, M. "Sensorless Sliding Mode Vector Control of Induction Motor Drives," International Journal of Power Electronics and Drive Systems, 2(3), 277, 2012.

[10] Yazdanpanah, R., Soltani, J., \& Markadeh, G. A. "Nonlinear Torque and Stator Flux Controller for Induction Motor Drive Based on Adaptive Input-output Feedback Linearization and Sliding Mode Control," Energy Conversion and management, 49(4), 541-550, 2008.

[11] Trong, T. N., "The Control Structure for DC Motor Based on the Flatness Control," International Journal of Power Electronics and Drive Systems (IJPEDS), 8(4), 1814-1821, 2017.

[12] Hagan, M. T., Demuth, H. B., \& Jesús, O. D., "An Introduction to the use of Neural Networks in Control Systems," International Journal of Robust and Nonlinear Control: IFAC-Affiliated Journal, 12(11), 959-985, 2002.

[13] Hunt K. J., Sbarbaro D., Zbikowski R., and Gawthrop P. J.: "Neural Networks for Control Systems-a Survey," Automatica, 28,(6), pp. 1083-1112, 1992.

[14] Hussain M. A.: "Review of the Applications of Neural Networks in Chemical Process Control-Simulation and Online Implementation," Arti- cial Intelligence in Engineering, 13, pp. 55-68, 1999.

[15] Denai M. A., Palis F., and Zeghbib, A.: "Modeling and Control of Non-linear Systems using Soft Computing Techniques," Applied Soft Computing, In Press, 2006.

[16] Douratsos, I., \& Gomm, J. B. (2007). Neural Network Based Model Reference Adaptive Control for Processes with Time Delay," International Journal of Information and Systems Sciences, 3(1), 161-179.

[17] Nguyen Trong, T., \& Nguyen Duc, M. "Sliding Surface in Consensus Problem of Multi-Agent Rigid Manipulators with Neural Network Controller,” Energies, 10(12), 2127, 2017. 